\title{
Expansion of a chromosomal repeat in Escherichia coli: roles of replication, repair, and recombination functions Anthony R Poteete
}

Address: Department of Molecular Genetics and Microbiology, University of Massachusetts Medical School, Worcester, MA, USA

Email: Anthony R Poteete - anthony.poteete@umassmed.edu

Published: 23 February 2009

BMC Molecular Biology 2009, 10:14 doi:10.1 186/1471-2199-10-14

This article is available from: http://www.biomedcentral.com/l47/-2/99//0/14

(c) 2009 Poteete; licensee BioMed Central Ltd.

This is an Open Access article distributed under the terms of the Creative Commons Attribution License (http://creativecommons.org/licenses/by/2.0), which permits unrestricted use, distribution, and reproduction in any medium, provided the original work is properly cited.
Received: II November 2008

Accepted: 23 February 2009

\begin{abstract}
Background: Previous studies of gene amplification in Escherichia coli have suggested that it occurs in two steps: duplication and expansion. Expansion is thought to result from homologous recombination between the repeated segments created by duplication. To explore the mechanism of expansion, a $7 \mathrm{kbp}$ duplication in the chromosome containing a leaky mutant version of the lac operon was constructed, and its expansion into an amplified array was studied.

Results: Under selection for lac function, colonies bearing multiple copies of the mutant lac operon appeared at a constant rate of approximately 4 to 5 per million cells plated per day, on days two through seven after plating. Expansion was not seen in a recA strain; null mutations in $\operatorname{recBCD}$ and ruvC reduced the rate 100 - and 10 -fold, respectively; a ruvC recG double mutant reduced the rate 1000 -fold. Expansion occurred at an increased rate in cells lacking dam, polA, rnhA, or uvrD functions. Null mutations of various other cellular recombination, repair, and stress response genes had little effect upon expansion. The red recombination genes of phage lambda could substitute for $r e c B C D$ in mediating expansion. In the red-substituted cells, expansion was only partially dependent upon recA function.
\end{abstract}

Conclusion: These observations are consistent with the idea that the expansion step of gene amplification is closely related, mechanistically, to interchromosomal homologous recombination events. They additionally provide support for recently described models of RecA-independent Redmediated recombination at replication forks.

\section{Background}

Expression of a chromosomal gene in Escherichia coli can be elevated by gene amplification. The mechanism of this amplification is thought to consist of two steps, duplication and expansion. Duplication is rare, largely recA-independent, and occurs between microhomologies in the chromosome as a replication accident. Expansion is frequent, recA-dependent, and thought to result from unequal crossing-over events between the duplicated segments [1-3].
Recent investigations of gene amplification in E. coli have focused on amplification of plasmid-borne genes. A phenotypically leaky F'-borne mutation, (lacIX13-lacZ), gives rise to $\mathrm{Lac}^{+}$revertants bearing amplified arrays of 40-80 copies of the lac region [4]. $\mathrm{Lac}^{+}$revertants of F'lac bearing the +1 frameshift allele (lacI33-lacZ), extensively employed in studies of adaptive mutation, consist mainly of one-base deletions in runs of iterated bases $[5,6]$, but clones bearing amplified arrays appear at a lower rate as well $[7,8]$. Properties of lac amplification have generally 
supported the duplication-expansion model. (i) An engineered duplication of the frameshift mutant lac locus amplifies at a greatly elevated frequency [9], as predicted by the idea that duplication is the rate-limiting step (and as had been seen in the case of chromosomal ampC [2]). (ii) Amplification is dependent upon $r e c B C D$ and ruvABC, as well as $r e c A$, indicating an important role for homologous recombination [10].

Expansion of a pre-existing repeat has also been studied primarily on plasmids. In one study, a pBR322 derivative was constructed with two directly repeated tetA genes, each bearing an inactivating mutation, but arranged in such a way that a single unequal crossover would generate an array of three copies, one of which was a functioning gene. In this system, expansion was reduced only five-fold in a recA mutant; expansion was elevated in strains bearing mutations in $d n a Q, d n a E$, dnaB, or dnaN [11].

Expansion of a pre-existing duplication was compared with amplification of a single copy of F'-borne (lacl33lacZ) in another study [10]. Expansion was found to be increased in a polA mutant, and unaffected by overexpression of $x o n A$, while amplification from a single copy was inhibited by both of these conditions. It was concluded that the amplification defects caused by the polA mutant and by xonA overproduction were in duplication, not expansion.

This study was undertaken to characterize expansion of a repeated sequence in the bacterial chromosome. A duplication of chromosomal (lacl33-lacZ) was constructed (Fig. 1). As expected, it expands at a high rate under selection for function. The effects of mutations in various recombination, replication, DNA repair, and stress response genes on expansion of the duplication were tested. The findings support the idea that expansion occurs via homologous recombination, and suggest as well that many of the recombination events leading to expansion take place at replication forks.

\section{Results}

An E. coli strain bearing a chromosomal duplication of the leaky (lacI33-lacZ) allele, when plated on minimal medium containing lactose as the only available carbon source, gives rise to approximately 1000 -fold more colonies, over the course of a week, than an otherwise isogenic strain bearing a single copy. As shown in Fig. 2, the colonies start appearing two days after plating, and accumulate at an average rate of approximately $4-5$ per million viable duplication-bearing cells plated per day, two to seven days after plating. Daily colony counts vary widely between independent cultures, as well as day-to-day on the same plate. This variation is considerably greater than that observed in experiments with single copy F'-borne

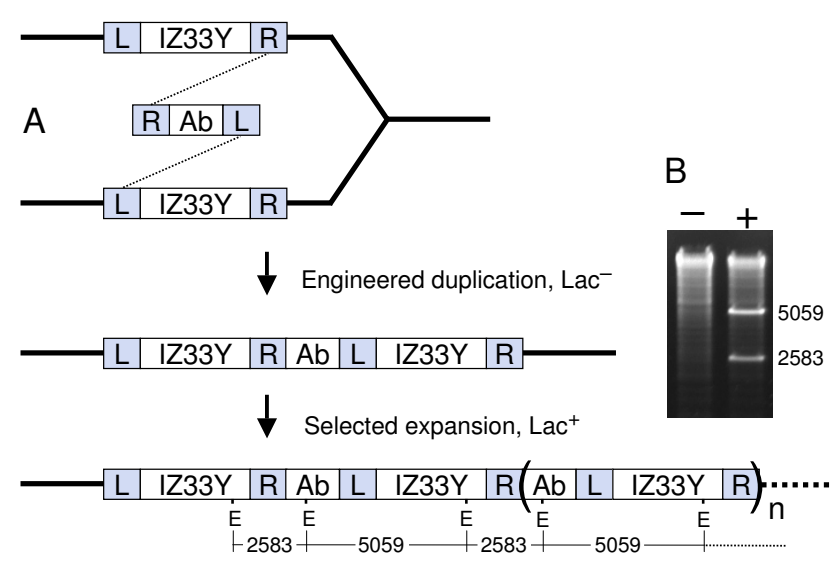

Figure I

Expansion of a chromosomal duplication. A. Chromosomal (lacl33-lacZ)-lacY [38] was duplicated by phage $\lambda$ Redmediated recombination with a linear DNA bearing homology-flanked antibiotic resistance marker Ab. A hypothetical mechanism by which the duplication could be generated, involving crossovers between the linear DNA and both copies of the replicating chromosomal target, is diagrammed [9]. The duplication was constructed with a tetracycline resistance element, which was later replaced with cat. Under selection for Lac function, the (laclZ33Y) $)_{2}$-cat duplication expands into multiple copies. $L$ and $R$ - chromosomal sequences flanking lac. E - EcoR I restriction sites. B. Multiple copies of the repeated sequence are seen as bands produced by EcoR I digestion of cellular DNA. Tests of two $\mathrm{Lac}^{+}$revertants, one without (-), and one with $(+)$ an expanded lac array, are shown as examples.

(lacI33-lacZ), in which the appearance of colonies after day two fits a Poisson distribution, implying that the mutations occurred after plating [12]. In contrast, the variation in colony counts seen in experiments with the chromosomal duplication strain indicate that most of the variation between cultures exists prior to plating (unpublished data). This observation is consistent with the hypothesis that each culture contains copy number variants which arise during growth, and that the probability of colony formation varies with copy number at the time of plating. Despite this variability, if 12 or more independent cultures are plated, and daily colony counts are averaged, the rate of accumulation is seen to be nearly constant, as reflected in the close fit of the data points to a straight line.

The $\mathrm{Lac}^{+}$colonies appearing in these experiments can arise either by expansion or by mutation. A strain bearing even a single chromosomal copy of the un-frameshifted lacIlacZ fusion grows well on lactose minimal medium. However, most or all of the excess $\mathrm{Lac}^{+}$colonies produced by the duplication-bearing strain contain expanded arrays of 


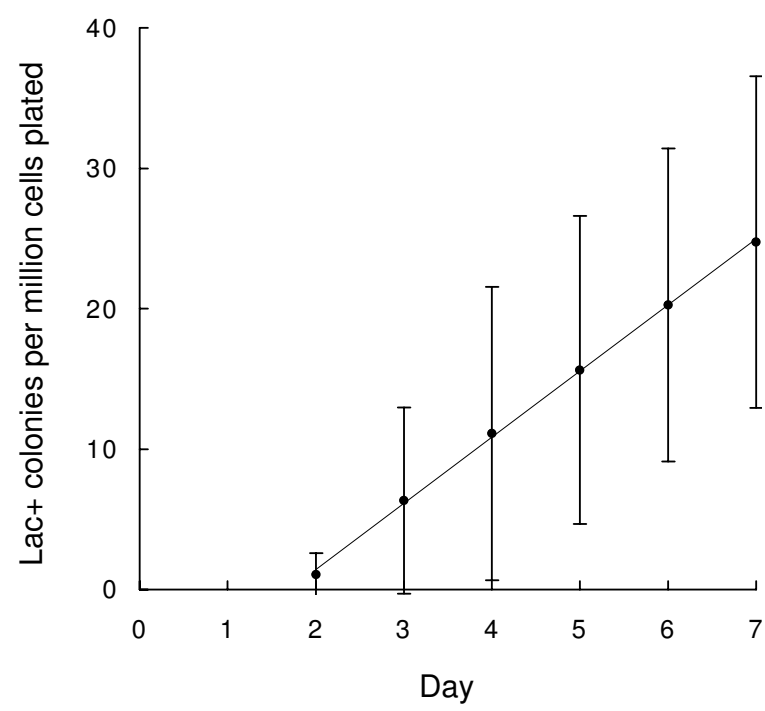

Figure 2

Kinetics of $\mathrm{Lac}^{+}$colony formation. Cultures of TPI004, an MGI 655 derivative bearing the (laclZ33Y $)_{2}$-cat duplication, were plated on lactose minimal agar. Data points represent mean daily colony counts from 19 independent cultures. Error bars represent I standard deviation. A least-squares linear regression curve is shown; its slope is $4.7 \mathrm{I}$ colonies per million viable chloramphenicol-resistant cells plated per day.

the structure diagrammed in Fig. 1. The amplified sequences in these clones are readily visualized as specific bands in restriction enzyme digests of total cellular DNA [4]. In tests of $28 \mathrm{Lac}^{+}$revertants from 22 cultures bearing the duplication, including four colonies which appeared on day two, all had expanded; none of 10 single-copy revertants contained amplified arrays (Table 1). Quantitation of total DNA, and DNA in the amplified bands, as described in the methods section, from $10 \mathrm{Lac}^{+}$clones, indicated a mean lac copy number of 72 , with a standard deviation of 17. Expansion of this magnitude would be expected to result in $\beta$-galactosidase production comparable to a single-copy un-frameshifted gene, as the frameshift mutation reduces $\beta$-galactosidase production 100 -fold [12]. The modest variability of lac copy number in the $\mathrm{Lac}^{+}$clones presumably reflects a sort of optimum or equilibrium, in which the benefit of more $\beta$-galactosidase is balanced by the cost of extra DNA in the chromosome (70 copies of the repeat make the chromosome roughly $10 \%$ larger). Cultures of the expanded-array variants contain unknown numbers of point-revertant lac genes. Under continued selection for lac function, it is possible that the population would eventually be taken over by revertants which, bearing a single good copy of lac, have the benefit of sufficient $\beta$-galactosidase without the cost of more DNA. However, it is likely that such a changeover would take many generations because the $\mathrm{Lac}^{+}$revertant has, if anything, only a small selective advantage - it does not have a noticeably faster growth rate on lactose minimal medium, for example. Hastings et al. [8] tested this idea, and found that amplified (lacI33lacZ) clones kept under selection for lac function do not form revertants readily.

The clones appearing as $\mathrm{Lac}^{+}$colonies acquire their ability to grow on lactose while under selection. Colonies restreaked on lactose minimal plates form colonies visible to the unaided eye in 24 hours or less, regardless of whether they were picked on day 2 or day 7 . Reversion and amplification of F'-borne (lacI33-lacZ) are adaptive, in that they occur only in the presence of lactose, not when the bacteria are simply starved $[8,13]$. The leakiness of the mutant allele is critical for adaptive mutation: residual lactose metabolism is enough to power the replication/recombination/repair processes involved, though not enough for cell division [12]. The experiment graphed in Fig. 3 shows that expansion of a chromosomal (lacI33lac $Z$ ) is similarly adaptive. Cultures were plated on minimal medium containing no available carbon source. Lac-

Table I: Expansion and survival tests

\begin{tabular}{lll}
\hline Genotype $^{\mathrm{a}}$ & $\begin{array}{l}\text { Lac clone expansion test }^{+} \\
\text {No. positive/no. tested }\end{array}$ & Survival on lactose minimal medium relative to wild type \\
\hline wild type & $28 / 28$ & 1 \\
recA & $0 / 10$ & $0.9 \pm 0.1$ \\
recBCD & $9 / 10$ & $0.9 \pm 0.1$ \\
ruvC & $10 / 10$ & $1.2 \pm 0.4$ \\
recG & $18 / 18$ & nd \\
ruvC recG & $6 / 14$ & $0.6 \pm 0.2$ \\
red+ & $10 / 10$ & nd \\
red+ recA & $8 / 10$ & nd \\
wild type single copy & $0 / 10$ & nd
\end{tabular}

a. See the legend to Figure 4 for a description of the strains tested for expansion. For the survival test, the (laclZ33Y $)_{2}$-cat duplication was replaced by a non-reverting lac deletion.

b. Means and standard errors are shown for three measurements. nd $=$ not determined. 


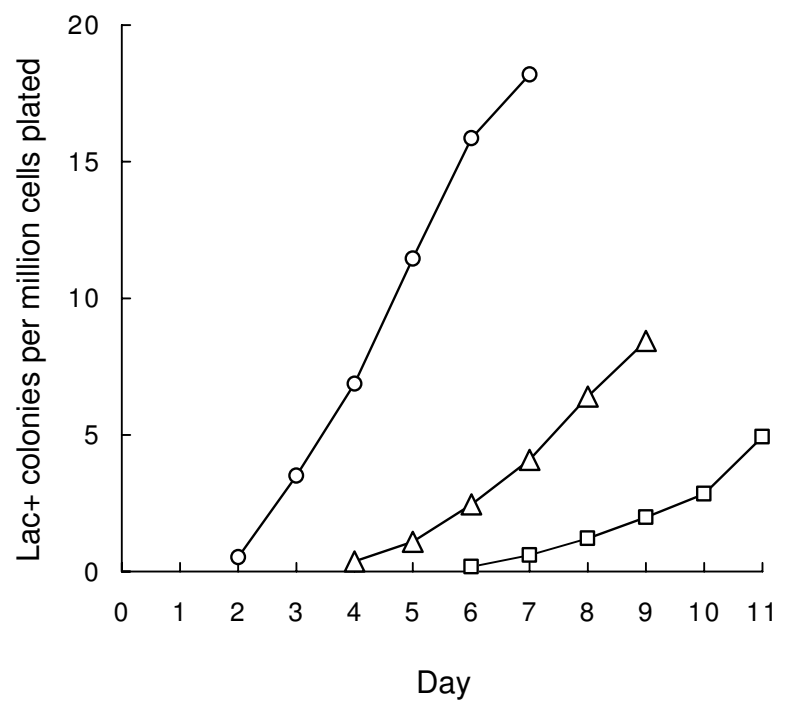

Figure 3

Adaptive nature of the expansion. Cultures of TPI004, an MGI 655 derivative bearing the (laclZ33Y) ${ }_{2}$-cat duplication, were plated on minimal agar in which lactose was made available as the only available carbon source, either at the time of plating (circles), or after two (triangles) or four (squares) days of incubation. Data points represent mean daily colony counts from 12 independent cultures. Error bars are omitted for clarity; as in Fig. 2, the standard deviations are comparable in magnitude to the means.

tose was added after two or four days by injection under the agar slab. There was no sudden burst of colonies appearing two or three days later, as would be expected if expanded lac arrays had accumulated during starvation. Rather, the kinetics of appearance of $\mathrm{Lac}^{+}$colonies resembled that seen in the cells initially plated on lactose, with a delay of either two or four days, and at declining rates, suggesting that the starving cells gradually lost their potential to expand.

Strains combining the duplication with mutations in DNA transaction genes were tested for $\mathrm{Lac}^{+}$colony formation (Fig. 4). A null mutation in recA reduced the rate nearly 1000-fold, nearly down to that of a strain bearing a single copy of (lacI33-lacZ) (labeled "sc" near the bottom of Fig. 4). The recA mutation had no significant effect in the single copy background. The strong recA dependence of expansion in this experiment contrasts with the weak recA dependence seen in a previous study of expansion of a plasmid-borne duplication [11], but is not surprising, for two reasons: (i) The duplicated segment in this study was much larger, and recA dependence tends to increase with increasing homology lengths [14,15]. (ii) The assay employed in the previous study required only a single recombination event, whereas becoming strongly $\mathrm{Lac}^{+}$by expansion of a chromosomal (lacI33-lacZ) duplication probably involves more than one recombination event.

Null mutations in $\mathrm{recBCD}$ and $\mathrm{ruvC}$ reduced $\mathrm{Lac}^{+}$colony formation 100-fold and 10-fold, respectively. Other mutations eliminating single recombination functions, $r e c F, r e c G, r e c N, \operatorname{rec} Q, r e c R$, and $r u v A B$, had little overall effect. A ruvC recG double mutant was also tested. Like the recA mutation, and as in other homologous recombination events [16], it generated $\mathrm{Lac}^{+}$colonies approximately 1000-fold less efficiently than wild type.

A disruption of the E. coli $y f g L$ gene was reported to confer a strong recombination/repair deficiency phenotype [17]. As shown in Fig. 4, however, a $\gamma f g L$ null mutation constructed for this study has little or no effect on expansion. It also confers no UV-sensitivity or transductional recombination phenotype, in either an MG1655 or an AB1157 strain background (not shown); others have found no recombination/repair phenotype associated with a $y f g L$ null as well [18].

To test the hypothesis that the deficiencies of recA, $\operatorname{rec} B C D, \operatorname{ruv} C$, and $\operatorname{ruv} C \operatorname{rec} G$ mutants in $\mathrm{Lac}^{+}$colony formation are due to their inability to expand the duplication, two alternative explanations were considered and ruled out. (i) $\mathrm{Lac}^{+}$revertants of these mutants could grow much more slowly than $\mathrm{Lac}^{+}$revertants of wild type. $\mathrm{Lac}^{+}$ colonies were restreaked on minimal lactose plates on the days they arose. In the cases of wild type, recA, recBCD, and $r u v C$, all of the $\mathrm{Lac}^{+}$clones formed colonies visible to the unaided eye by 24 hours after restreaking, independent of the day on which they arose. In the case of the ruvC recG double mutant, none of the 8 tested $\mathrm{Lac}^{+}$revertants formed visible colonies by 24 hours, but all did so by 48 hours. However, counting the ruvC recG colonies after 8 days instead of 7 only increased the median from 0.029 to 0.056 colonies per million viable cells plated (data not shown). Thus, slow growth of revertants can account for only a small part of the deficiencies of the recombination mutants in forming $\mathrm{Lac}^{+}$colonies. (ii) The mutants could be proficient at expansion, but deficient at survival on the selection plates. Survival of the mutants on lactose minimal medium was tested as described in the methods section. The results (Table 1) indicate that none of the mutants has a substantial survival defect relative to wild type.

The $\mathrm{Lac}^{+}$revertants of the deficient mutants consist of varying populations of expanded and mutated clones (Table $1)$. In the case of $r e c A$, none of 10 tested clones had an expanded lac array. The frequency of expanded arrays among recBCD revertants was 9 out of 10 ; ruv $C$ was 10 out of 10 , recG was 18 out of 18 , and $\operatorname{ruv} C$ recG was 6 out of 14. 


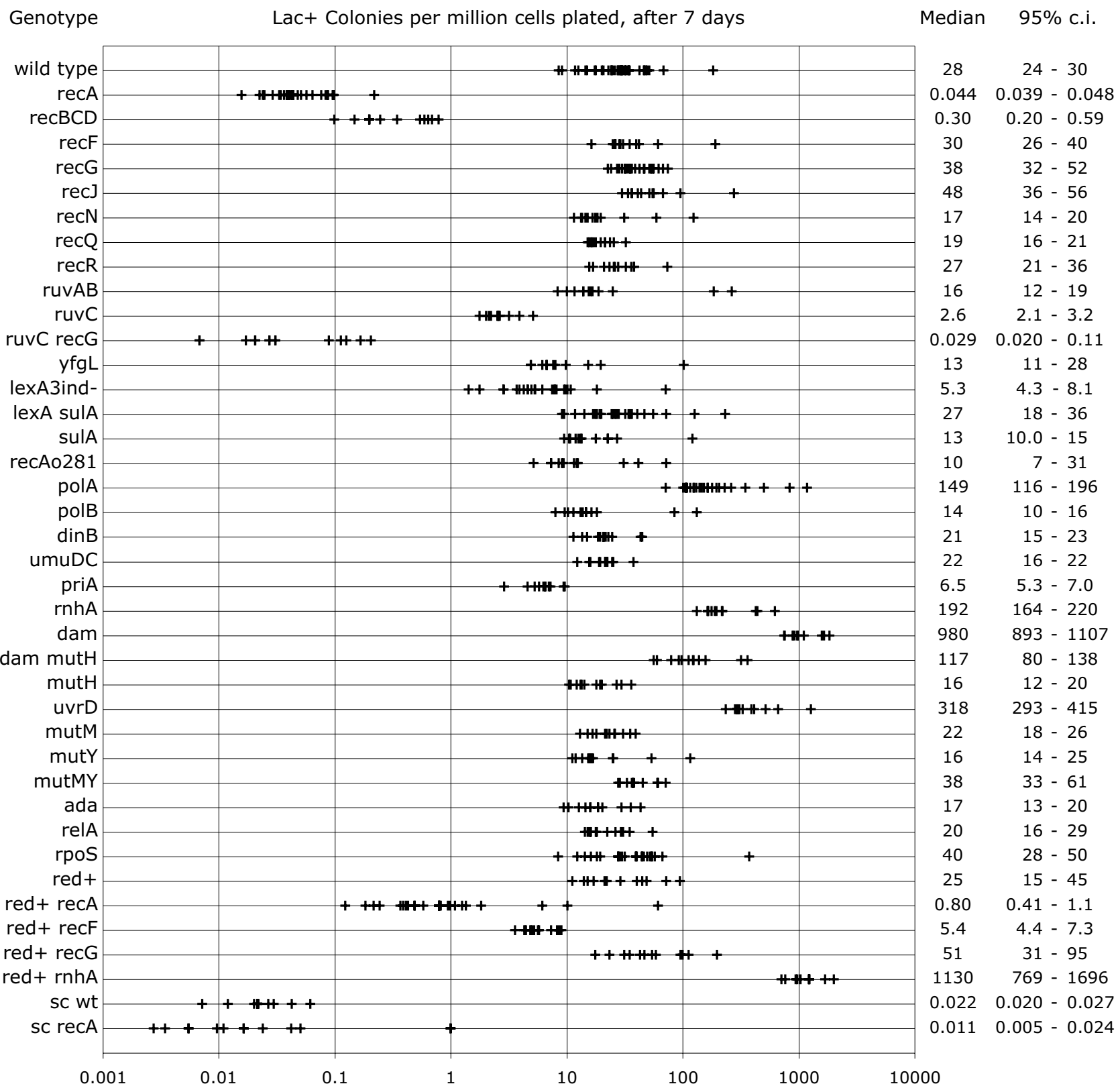

\section{Figure 4}

Roles of replication, repair, and recombination functions in expansion. Multiple independent cultures of the indicated genotype were plated on lactose minimal medium. Strains are all MG I655 derivatives. All except the ones labeled "sc" (for single copy) bear the the (laclZ33Y $)_{2}$-cat duplication. Strains labeled "red+" bear the phage $\lambda$ red recombination genes, which replace the recC-ptr-recB-recD gene cluster in the $E$. coli chromosome. Except for the red substitution, the lexA alleles, and the recAo28I operator mutation, all the alleles are nulls made by substituting an antibiotic resistance element for the coding sequence of the gene.

The $\mathrm{Lac}^{+}$reversion phenotype of a recG null mutant is more complex than the data in Fig. 4 suggest. The mutant strain's rate of colony formation tends to increase sharply late in the experiment, with the new colonies tending to appear as satellites of older colonies (not shown). Factors influencing the timing and extent of this satellite-based population explosion include plating density, but are otherwise unknown. The recG mutant data shown in Fig. 4 are from selected experiments, in which the plating density was low, and satellitism was not as strongly evident as in 
other experiments. Satellitism of this sort suggests that something produced by the older colonies on the plate stimulates recombination, perhaps via a genotoxic effect. It is consistent with the finding that overexpression of rec $G$ protects E. coli against weak organic acids [19].

The effects on expansion of varying RecA activity were tested by plating mutants affecting recA expression. The uninducible lexA3 mutation has been reported to reduce the frequency of a number of different homologous recombination events [20]; it also reduced $\mathrm{Lac}^{+}$colony formation approximately five-fold. The SOS-constitutive lexA71::Tn5 mutation (in a sulA null background, to suppress its lethality) had no significant effect; neither did the recAo281 operator constitutive allele.

A number of replication genes were tested for roles in expansion. A null mutation in polA increased the rate of expansion 5-fold; null mutations in the other non-essential DNA polymerase-encoding genes polB, $\operatorname{din} B$, and $u m u D C$, had little or no effect. Loss of the replication restart function priA caused a small decrease in expansion efficiency, while loss of $r n h A$ caused a nearly 7 -fold increase.

A strain lacking dam function exhibited a 35-fold elevated rate of $\mathrm{Lac}^{+}$colony formation. Apparently, part of this elevated rate is due to the double-strand breaks which occur as the result of mis-directed mismatch repair in dam mutants [21,22]. As shown in Fig. 4, a dam mutH double mutant exhibited an intermediate rate between those of wild type and the dam single mutant. The mutH null allele by itself had little or no effect.

Other DNA repair functions were tested for effects on expansion as well. A uvrD null mutant formed $\mathrm{Lac}^{+}$colonies at a 10-fold elevated rate, while mutM, mutY, a mutM mutY double, and an ada null mutation had no significant effects.

The question of whether expansion of a chromosomal repeat occurs as part of a stress response, like amplification starting from a single episomal copy [23], was explored by testing null mutations in rpoS and relA. As shown in Fig. 4, these mutations had little or no effect on $\mathrm{Lac}^{+}$colony formation.

Expansion mediated by the Red recombination system of phage $\lambda$ was studied in a series of strains in which the phage red genes replace the $r e c C$-ptr-recB-rec $D$ gene cluster in the E. coli chromosome (designated "red+" in Fig. 4). Replacing RecBCD with Red has no effect on the rate of $\mathrm{Lac}^{+}$colony formation, but it changes the extent to which expansion is dependent upon other recombination functions. In the red-substituted background, a recA null mutation reduces $\mathrm{Lac}^{+}$colony formation only 35-fold. Among the rec $A$ revertants, 8 of 10 that were tested contained expanded lac arrays (Table 1), showing that Red, unlike RecBCD, can promote expansion in the absence of RecA. Red-mediated expansion is reduced by a recF null mutation, and elevated slightly by a recG null; these mutant effects are seen in Red-mediated gene replacement events as well [24]. The rnhA null mutation has a stronger effect in the red-substituted background than in wild type, elevating the rate of expansion 45-fold.

\section{Discussion and conclusion}

The genetic requirements of homologous recombination in $E$. coli vary with the particular event examined, but some features of chromosomal events are nearly general: dependence on recA and $\operatorname{rec} B C D$, and mild or no dependence upon a variety of other recombination functions whose roles are revealed mainly in the absence of $r e c B C D$ function [14]. Expansion by duplicated chromosomal (lacI33-lacZ) fits this general pattern. Similarly, mutations with known hyper-rec phenotypes - polA, dam, uvrD, and $r n h A[20,25,26]$ - also cause an elevated rate of expansion. These observations support the idea that expansion is best understood as a homologous recombination event or series of events.

The Red recombination system of phage $\lambda$ promotes RecA-independent recombination between chromosomes if at least one of the chromosomes is replicating, and RecA-dependent recombination between non-replicating chromosomes [27-30]. The RecA-independent expansion seen in red-substituted bacteria suggests that at least some of the Red-mediated recombination events involved in expansion take place at replication forks [30,31].

The involvement of replication in recombination events leading to expansion is additionally suggested by the increased rates of expansion of the rnhA and dam mutants. Replication in both these mutants escapes cell cycle regulation. In an $r n h A$ mutant, unsynchronized DNA replication initiates at multiple sites in the chromosome [32]. In a dam mutant, initiation is confined to oriC but is not regulated [33]. Elevated recombination frequencies in both of these mutants may be due, at least in part, to an increased occurrence of double strand breaks. Both exhibit greatly reduced viability in the absence of RecBCD function, possibly because RecBCD is needed to repair the excess double strand breaks $[34,35]$. Eliminating the mismatch repair endonuclease MutH in a dam mutant prevents the double strand breaks which result from misdirected mismatch repair, but does not bring expansion down to the wild type level; unregulated replication itself is a possible cause of the residual excess expansion in the dam mutH double mutant. The mechanism by which improperly regulated replication forks provoke more recombination events than normally regulated replication forks is unknown, but there are a number of possible 
Table 2: Strains

\begin{tabular}{|c|c|c|}
\hline Strain & Relevant Genotype & Reference, source, or construction \\
\hline FC69I & $\operatorname{laclZ33^{a}}$ & {$[38]$} \\
\hline GM3819 & $\operatorname{dam} \Delta \mathrm{kan}$ & M. Marinus \\
\hline GM829I & polA $\Delta$ frt-kan/F' polA+ camR & M. Marinus; polA allele [39] \\
\hline MGI655 & wild type & \\
\hline MVII32 & srl300::TnI0 recAo28I & M. Volkert \\
\hline MVII 54 & $\operatorname{lex} \mathrm{A} 3$ & M. Volkert \\
\hline MV2IO4 & lexA7|::Tn5 & M. Volkert \\
\hline TP538 & $\operatorname{recG} \Delta$ tet & {$[40]$} \\
\hline TP539 & recG $\Delta$ kan & [40] \\
\hline TP540 & $\operatorname{ruv} A B \Delta$ tet & {$[40]$} \\
\hline TP547 & red-cat ${ }^{b}$ & derivative of KM32 [4I] \\
\hline TP577 & $\operatorname{recF} \Delta$ tet & {$[40]$} \\
\hline TP605 & sulA $\Delta$ tet & {$[40]$} \\
\hline TP643 & recQ $\Delta$ tet & [24] \\
\hline TP645 & recR $\Delta$ tet & {$[24]$} \\
\hline TP662 & sulA $\Delta$ kan & substitution of Tn903 aph for TnI0 tetRA in sulA $\Delta$ tet [40] \\
\hline TP664 & $\operatorname{recN} \Delta$ tet & {$[42]$} \\
\hline TP730 & red-cat laclZ33 & FC69I × PI•TP547 \\
\hline TP732 & red-pae-clb laclZ33 & TP730 $\times$ PTP822 linear [4I] \\
\hline TP796 & recA $\Delta$ tet & [43] \\
\hline TP797 & ruvC $\Delta$ tet & [43] \\
\hline TP798 & red-cat & [36] \\
\hline TP832 & red-amp $p^{b}$ & [36] \\
\hline TP838 & recBCD $\Delta$ tet & [43] \\
\hline TP872 & red-amp lac $\Delta$ cat & TP832 $\times$ cat 15,16 pcr of Tn9c \\
\hline TP889 & lac $\Delta$ cat & MGI655 × PI•TP872 \\
\hline TP922 & red-pae-clb $(\text { laclZ33Y })_{2}$-tet-oriR6Kãd & TP732 $\times$ PTPI06I lineare \\
\hline TP929 & $(\text { laclZ33Y })_{2}$-tet-oriR6Kgamma & TP889 $\times$ PI•TP922 \\
\hline TP942 & red-pae-cl $(\text { laclZ33Y })_{2}$-cat & TP922 $\times$ Tocl, 2 pcr of Tn9 \\
\hline TP997 & galK::aacCl067 & {$[44]$} \\
\hline TPI000 & $\operatorname{din} B \Delta$ tet & TP798 $\times \operatorname{din} 7,8$ pcr of Tnl0 \\
\hline TPI003 & $\operatorname{lac} \Delta \mathrm{spc}$ & $\begin{array}{l}\text { TP889 was transduced with a PI lysate of an unnamed intermediate strain, TP798 } \times \text { LAT2,3 } \\
\text { pcr of Tn2I aadA }\end{array}$ \\
\hline TPI004 & $(\text { laclZ33Y })_{2}$-cat & TP929 × PI·TP942 \\
\hline TPI005 & $(\text { laclZ33Y })_{2}$-cat dinB $\Delta$ tet & TPI004 × PI·TPI000 \\
\hline TPI006 & $(\text { laclZ33Y })_{2}$-cat recA $\Delta$ tet & TPI004 × PI•TP796 \\
\hline TPI007 & $(\text { laclZ33Y })_{2}$-cat recF $\Delta$ tet & TPI004 × PI•TP577 \\
\hline TPI008 & $(\text { laclZ33Y })_{2}$-cat recG $\Delta$ tet & TPI004 × PI•TP538 \\
\hline TPI009 & $(\text { laclZ33Y })_{2}$-cat recN $\Delta$ tet & TPI004 × PI•TP664 \\
\hline TPIOII & $(\text { laclZ33Y })_{2}$-cat recQ $\Delta$ tet & TPI004 × PI•TP643 \\
\hline TPIOI2 & $(\text { laclZ33Y })_{2}$-cat recBCD $\Delta$ tet & TPI004 $\times$ PI•TP838 \\
\hline TPI0I4 & $(\text { laclZ33Y })_{2}$-cat ruvAB $\Delta$ tet & TPI004 × PI•TP540 \\
\hline TPI0I5 & $(\text { laclZ33Y })_{2}$-cat ruvC $\Delta$ tet & TPI004 × PI•TP797 \\
\hline TPI020 & $(\text { laclZ33Y })_{2}$-cat recR $\Delta$ tet & TPI004 × PI•TP645 \\
\hline TPI022 & laclZ33 & Spontaneous chloramphenicol-sensitive TPI004 derivative \\
\hline TPI03। & red-cat rec $\Delta$ tet & TP798 $\times$ recjI,2 pcr of Tnl0 \\
\hline TPI032 & red-cat $\mathrm{rhnA} \Delta$ tet & TP798 $\times$ rnhAI,2 pcr of Tnl0 \\
\hline TPI033 & red-cat polB $\Delta$ tet & TP798 $\times$ polBI,2 pcr of TnI0 \\
\hline TPI034 & $(\text { laclZ33Y })_{2}$-cat recj $\Delta$ tet & TPI004 × PI•TPI03I \\
\hline TPI035 & $(\text { laclZ33Y })_{2}$-cat rhnA $\Delta$ tet & TPI004 × PI•TPI032 \\
\hline TPI036 & $(\text { laclZ33Y })_{2}$-cat polB $\Delta$ tet & TPI004 × PI•TPI033 \\
\hline TPI038 & red-amp (laclZ33Y $)_{2}$-cat & TP832 $\times$ PI•TP942 \\
\hline TPI042 & $(\text { laclZ33Y })_{2}$-cat dam $\Delta$ kan & TPI004 × PI·GM3819 \\
\hline TPI043 & $(\text { laclZ33Y })_{2}$-cat sulA $\Delta$ tet & TPI004 × PI·TP605 \\
\hline TPI047 & $(\text { laclZ33Y })_{2}$-cat urrD $\Delta$ tet & TPI004/PKM208 × uvrDI,2 pcr of TnI0 \\
\hline TPI048 & $(\text { laclZ33Y })_{2}$-cat sulA $\Delta$ tet lexA7I::Tn5 & TPI043 $\times \mathrm{PI} \cdot \mathrm{MV} 2104$ \\
\hline TPI049 & $(\text { laclZ33Y })_{2}$-cat umuDC $\Delta$ tet & TPI004/pKM208 [45] $\times$ umDCI,2 pcr of Tnl0 \\
\hline TPI050 & $(\text { laclZ33Y })_{2}$-cat malF $\Delta s p c$ & TPI004/pKM208 × malFI,2 pcr of TP997 \\
\hline TPI05I & $(\text { laclZ33Y })_{2}$-cat rpoS $\Delta$ tet & TPI004/pKM208 $\times$ rpoSI,2 pcr of Tnl0 \\
\hline TPI053 & $(\text { laclZ33Y })_{2}$-cat ada $\Delta$ kan & TPI004/PKM208 × ADAI,2 pcr of Tn903 aph \\
\hline
\end{tabular}


Table 2: Strains (Continued)

\begin{tabular}{|c|c|c|}
\hline TPI054 & $(\text { laclZ33Y })_{2}$-cat priA $\Delta$ kan & TPI004/pKM208 × priAI,2 pcr of Tn903 aph \\
\hline TPI055 & $(\text { laclZ33Y })_{2}$-cat relA $\Delta$ kan & TPI004/pKM208 $\times$ relAI,2 pcr of Tn903 aph \\
\hline TPI056 & $(\text { laclZ33Y })_{2}$-cat sulA $\Delta$ tet priA $\Delta$ kan & TPI043 × PI•1054 \\
\hline TPI057 & red-amp (laclZ33Y $)_{2}$-cat recA $\Delta$ tet & TPI038 × PI•796 \\
\hline TPI058 & red-amp (laclZ33Y $)_{2}$-cat recF $\Delta$ tet & TPI038 × PI.577 \\
\hline TPI059 & red-amp (laclZ33Y $)_{2}$-cat recG $\Delta$ tet & TPI038 × PI.538 \\
\hline TPI06I & red-amp (laclZ33Y $)_{2}$-cat rnhA $\Delta$ tet & TPI038 $\times$ PI.1035 \\
\hline TPI062 & laclZ33 recA $\Delta$ tet & TPI022 $\times \mathrm{PI} \cdot 796$ \\
\hline TPI063 & $(\text { laclZ33Y })_{2}$-cat recA $\Delta$ kan & TPI004/pKM208 × recA3,4 pcr of Tn903 aph \\
\hline TPI064 & $(\text { laclZ33Y })_{2}$-cat mutH $\Delta$ tet & TPI004/pKM208 × mutHI,2 pcr of 1016 \\
\hline TPI065 & $(\text { laclZ33Y })_{2}$-cat mutM $\Delta$ tet & TPI004/pKM208 $\times$ mutMI, 2 pcr of Tnl0 \\
\hline TPI066 & $(\text { laclZ33Y })_{2}$-cat mutY $\Delta$ kan & TPI004/pKM208 × mutYI,2 pcr of Tn903 aph \\
\hline TPI069 & $(\text { laclZ33Y })_{2}$-cat mutM $\Delta$ tet mutY $\Delta$ kan & TPI065 × PITPI066 \\
\hline TPI080 & lac $\Delta$ cat $\operatorname{rec} A \Delta$ tet & TP889 × PI•TP796 \\
\hline TPI08I & lac $\Delta$ cat $\operatorname{recBCD} \Delta$ tet & TP889 $\times \mathrm{PI} \cdot$ TP838 \\
\hline TPI082 & lac $\Delta$ cat ruvC $\Delta$ tet & TP889 $\times$ PI•TP797 \\
\hline TPI086 & $(\text { laclZ33Y })_{2}$-cat polA $\Delta$ frt-kan & TPI004 × PI•JW3835 \\
\hline TPI089 & $(\text { laclZ33Y })_{2}$-cat yfgL $\Delta$ kan & TPI004/pKM208 $\times$ yfgLI,2 pcr of Tn903 aph \\
\hline TPI090 & $(\text { laclZ33Y })_{2}$-cat lexA3 & TPI050 × PI•MVII54, selection for $\mathrm{Mal}^{+}$, screen for UV-sensitivity \\
\hline TPI09| & $(\text { laclZ33Y })_{2}$-cat dam $\Delta$ kan mutH $\Delta$ tet & TPI064 × PI·GM38I9 \\
\hline TPI095 & $(\text { laclZ33Y })_{2}$-cat srl300::Tn 10 recAo28I & TPI063 $\times \mathrm{PI} \cdot \mathrm{MVII} 32$ \\
\hline TPI098 & $(\text { laclZ33Y })_{2}$-cat ruvC $\Delta$ tet recG $\Delta$ kan & TPI015 × PI•TP539 \\
\hline TPI099 & $(\text { laclZ33Y })_{2}$-cat recAo28I & $\begin{array}{l}\text { TPI } 095 \times \mathrm{PI} \cdot \mathrm{TPI} 063 \text {, selection for Srl+, screen for kanamycin sensitivity and the Sph site } \\
\text { created by the recA028I mutation }[46]\end{array}$ \\
\hline
\end{tabular}

\section{Notes}

a. The abbreviation laclZ33 is used to denote the triple mutant (lacl33-lacZ) bearing the lacle promoter-up mutation in the lacl promoter, a 2 I2-bp deletion fusing lacl to lac $Z$, and a +1 frameshift mutation in the lacl sequences near the fusion junction [ 13 ].

b. Strains designated "red" bear the phage $\lambda$ red recombination genes, which replace the recC-ptr-recB-recD gene cluster in the $E$. coli chromosome.

c. Primers used in polymerase chain reactions to generate antibiotic cassettes for gene replacements are described in Table 3 .

d. Strains designated "(laclZ33Y $)_{2}$-" bear a duplication of laclZ33 and lacY, with the genetic element listed after the hyphen (tet-oriR6Kgamma or cat) inserted between the two copies.

e. Details of this construction are given in the text.

explanations. They might be more prone to breaking down or to running into each other, or just more numerous in the cell.

\section{Methods \\ Duplication of (lacl33-lacZ)-lacY}

Plasmid pTP1029 [36] is a vector containing the tetRA genes from $\mathrm{Tn} 10$ and the pir-dependent replication origin R6Korigamma. pTP1060 was made by ligating a synthetic DNA made from two oligodeoxyribonucleotides, GATCCAGGTTCTTTGAGCTCTTTGGCGGCCGC and GATCGC GGCCGCCAAAGAGCTCAAAGAACCTG, into the BamHI site of pTP1029. pTP1016 [36] contains E. coli sequences which normally flank the lacI and lacY genes; in the plasmid, they flank the cat gene. pTP1027 [36] and pTP1049 [37] contain the same flank sequences as pTP1016, with wild type lacIZY and (lacI33-lacZ)-lacY, respectively, between them. pTP1061 was made by ligating the catwith-lac-flanks cassette of pTP1016 into the NotI site of pTP1060. A strain bearing a duplication of the chromosomal segment containing (lacI33-lacZ)-lacY was constructed by electroporating strain TP890 with BsrG1- and Sph1-digested plasmid pTP1061, and selecting for tetracycline resistance (see Fig. 1). The tet-origamma insert between the duplicated lac copies was replaced by cat via recombination with a linear DNA generated by PCR with a Tn9-containing strain as template, and primers GATCCCGCGGAATAACATCATTTGGTGACGAAATAA C TAAATGAGACGTTGATCGGCACG and CCACGATGC GT CCGGCGTAGAGGATCTGAAGATCAGCA GTATTCAGGC GTAGCACCAGGC. The presence of duplicated segments in bacterial chromosomes was verified by the use of PCR with divergent primers, as described [36]. Other strain construction details are given in Tables 2 and 3.

\section{Plating methods}

Strains to be tested for reversion to $\mathrm{Lac}^{+}$were grown to saturation in $\mathrm{M} 90.1 \%$ glycerol minimal medium at $37^{\circ} \mathrm{C}$, and plated on $\mathrm{M} 90.1 \%$ lactose plates at $37^{\circ} \mathrm{C}$. M9 minimal media, supplemented with thiamine at $5 \mu \mathrm{g} / \mathrm{ml}$, were as described [7]. In most cases, viable duplication-positive titers were determined by plating on LB agar supplemented with chloramphenicol at $10 \mu \mathrm{g} / \mathrm{ml}$, which permits colony formation only by bacteria retaining the cat gene between the duplicated segments. Strains with poor viability in rich media ( $r u v C$ recG, polA, priA) were titered on M9 glucose plates; retention of the duplication in these cases was assessed by testing the chloramphenicol resistance of individual colonies from the titer plates. Lactose minimal plates were inoculated with $1-2 \times 10^{9}$ cells, 
Table 3: Primers

\begin{tabular}{|c|c|}
\hline Primer & Sequence \\
\hline ADAI & GATTATGAAAAAAGCCACATGCTTAACTGACGATCAACGCACGTTGTGTCTCAAAATCTC \\
\hline ADA2 & CTCCTCATTTTCAGCTTCGCGGCGCAGCAGTTGCGCTTTACAACCAATTAACCAATTCTG \\
\hline cat 15 & TCTGGTGGCCGGAAGGCGAAGCGGCATGCATTTACGTTGAATGAGACGTTGATCGGCACG \\
\hline cat 16 & AGAGTACATCTCGCCGTTTTTTTCTCAATTCATGGTGTACAATTCAGGCGTAGCACCAGGC \\
\hline $\operatorname{din} 7$ & GCTGGATAAGCAGCAGGTGCTTTCGCAGCGAACGCGTTAACTCGACATCTTGGTTACCGT \\
\hline $\operatorname{din} 8$ & ACCAGTTGTCTTTCCATTTGCGGGTCAAGCAACGTCACATCGCGGAATAACATCATTTGG \\
\hline LAT2 & AAGAAAGCCTGACTGGCGGTTAAATTGCCAACGCTTATTATTATTTGCCGACTACCTTGG \\
\hline LAT3 & GACGGGTTGTTACTCGCTCACATTTAATGTTGATGAAAGCAAACGGATGAAGGCACGAA \\
\hline malFI & GTCCTGGAATGAGGAAGAACCCCATGGATGTCATTAAAAAAAACGGATGAAGGCACGAA \\
\hline malF2 & CCCTTAATCAAACTTCATTCGCGTGGCTTTCAGGTTCACTTTATTTGCCGACTACCTTGG \\
\hline mutHI & TTTTTTAATCAAGGTATCATGACATGTCCCAACCTCGCCCCTCGACATCTTGGTTACCGT \\
\hline mutH2 & GCGATGGCTACTGGATCAGAAAATGACGGGCCAGTAGTGCCGCGGAATAACATCATTTGG \\
\hline mutMI & GCATCTGTTCATTCCTGGAGATGCTATGCCTGAATTACCCCTCGACATCTTGGTTACCGT \\
\hline mutM2 & TCCGGCGCGCATGAATTACTTCTGGCACTGCCGACAATAACGCGGAATAACATCATTTGG \\
\hline mutYI & CAACAGTGAATTCGGTGACCATGCAAGCGTCGCAATTTTCACGTTGTGTCTCAAAATCTC \\
\hline mutY2 & CTTTATCGACTCACGCGCTAAACCGGCGCGCCAGTGCGTACAACCAATTAACCAATTCTG \\
\hline polBI & GTGGCGCAGGCAGGTTTTATCTTAACCCGACACTGGCGGGCTCGACATCTTGGTTACCGT \\
\hline polB2 & AGTGGTGAACGTTGGTAGTCCAGCGGCTCCGGGCCGTTGGCGCGGAATAACATCATTTGG \\
\hline priAl & GATGCTATGCCCGTTGCCCACGTTGCCTTGCCCGTTCCGCACGTTGTGTCTCAAAATCTC \\
\hline priA2 & GGAATCCGGTATTGTATTGATGAGCGCCAGCGTACCGTTACAACCAATTAACCAATTCTG \\
\hline recA3 & TTACCCGGCATGACAGGAGTAAAAATGGCTATCGACGAACACGTTGTGTCTCAAAATCTC \\
\hline recA4 & CCCTTGTGTATCAAACAAGACGATTAAAAATCTTCGTTTACAACCAATTAACCAATTCTG \\
\hline recjl & ACAGATACAACTTCGTCGCCGTGAAGTCGATGAAACGGCACTCGACATCTTGGTTACCGT \\
\hline recj2 & GCAGTGGACCGCCGCCGACCGGTTCGACCATCACCTTCAACGCGGAATAACATCATTTGG \\
\hline relAI & GGACGATGGTTGCGGTAAGAAGTGCACATATCAATAAGGCACGTTGTGTCTCAAAATCTC \\
\hline relA2 & GGTCATGTCGATGGTCGCCAGTTGCTGTTTGGTGTCGCTACAACCAATTAACCAATTCTG \\
\hline rnhAl & GGCAATCCAGGACCTGGGGGTTACGGCGCTATTTTACGCTCTCGACATCTTGGTTACCGT \\
\hline rnhA2 & GCCGCGGCACGAGCCAGTTCATCACAGCGTTCGTTTTCCGCGCGGAATAACATCATTTGG \\
\hline rpoSI & CGGGTAGGAGCCACCTTATGAGTCAGAATACGCTGAAAGTCTCGACATCTTGGTTACCGT \\
\hline rpos2 & ССTTTCTGACAGATGCTTACTTACTCGCGGAACAGCGCTTCGCGGAATAACATCATTTGG \\
\hline Tocl & GATCCCGCGGAATAACATCATTTGGTGACGAAATAACTAAATGAGACGTTGATCGGCACG \\
\hline Toc2 & CCACGATGCGTCCGGCGTAGAGGATCTGAAGATCAGCAGTATTCAGGCGTAGCACCAGGC \\
\hline umDCl & CAGATTATTATGTTGTTTATCAAGCCTGCGGATCTCCGCGCTCGACATCTTGGTTACCGT \\
\hline umDC2 & CCTGCCGCTATATTTATTTGACCCTCAGTAAATCAGAACTCGCGGAATAACATCATTTGG \\
\hline uvrDI & AACCTATTTTTACGCGGCGGTGCCAATGGACGTTTCTTACCTCGACATCTTGGTTACCGT \\
\hline uvrD2 & CTGGCCCTGAAATGCCACCTGCAAACGGCTATGCTCACCGCGCGGAATAACATCATTTGG \\
\hline yfgLI & TCTGAGAGGGACCCGATGCAATTGCGTAAATTACTGCTGCACGTTGTGTCTCAAAATCTC \\
\hline yfgL2 & GCCGTCAGCGGCAACCGGTTCAGTCTGGAAACCGGAACTACAACCAATTAACCAATTCTG \\
\hline
\end{tabular}

either of the strain to be tested by itself, or, in most cases, of the strain to be tested plus a non-reverting, lac deletionbearing scavenger strain [13]. The rates of appearance of $\mathrm{Lac}^{+}$colonies shown in Figures 2, 3, and 4 are calculated as $\mathrm{Lac}^{+}$colonies per million chloramphenicol-resistant viable cells plated (or per million viable cells, in the cases of the single copy strains).

\section{Expansion test}

Revertant colonies appearing on the M9 lactose plates were streaked on M9 lactose plates, which were incubated at $37^{\circ} \mathrm{C}$ until visible colonies formed. Heavy inocula constituting the bulk of the growth from the streaks were then scraped from the plate and grown to saturation in $5 \mathrm{ml} \mathrm{M9}$ $0.1 \%$ lactose minimal medium at $37^{\circ} \mathrm{C}$. (Some of the revertant colonies tested, in the wild type background only, were inoculated directly from the selection plates into liquid lactose minimal medium; but all revertants in the wild type background tested positive for expanded arrays, regardless of the variation in culture methods). DNA was extracted by the use of a procedure involving freezing and thawing, lysozyme digestion, extraction with a phenol/chloroform/isoamyl alcohol mixture, extraction with ether, and precipitation with ethanol [30]. Portions were digested with EcoR1 and RNase, and subjected to electrophoresis in an agarose gel, followed by ethidium bromide staining. For quantitation of the repeat-specific bands, standards consisting of HindIII-digested phage lambda DNA of known concentration were included in the gel, in separate lanes. Total DNA in the sample was quantitated by spotting RNase-treated samples on an agarose slab containing ethidium bromide at $1 \mu \mathrm{g} / \mathrm{ml}$, along 
with standards of known concentration. Band and spot intensities were measured by the use of digital photography and Kodak 1D software.

\section{Survival test}

Mutations to be tested for their effects on survival on lactose minimal medium were crossed into a strain bearing a deletion of the lac operon. Cultures were grown to saturation in $\mathrm{M} 90.1 \%$ glycerol as in the $\mathrm{Lac}^{+}$reversion test, and deposited on the surfaces of $0.6 \mathrm{ml} \mathrm{M} 9$ lactose agar plugs at the bottom of $12 \times 75 \mathrm{~mm}$ plastic tubes, at approximately the same plating density (relative to volume of medium) as the bacteria in the $\mathrm{Lac}^{+}$reversion test. The tubes were incubated at $37^{\circ} \mathrm{C}$. Cells were suspended by vortexing in $2 \mathrm{ml}$ of buffer, and titered on $\mathrm{M} 9$ glucose plates, on days 0 and 7 . The ratio of titer on day 7 to titer on day 0 for the wild type control was $0.83 \pm 0.12$ (mean \pm standard error from six measurements).

\section{Abbreviations}

Kbp: kilobasepair.

\section{Acknowledgements}

I thank Patricia Foster, Michael Volkert, Martin Marinus, and Susan Lovett for helpful discussions; Michael Volkert and Martin Marinus for strains; and Rosemary Proff, Dery Miller, Shilpa Nadimpalli, Bach Nguyen, Juliet Zhang, Aiden Galarza, and Matthew Lim for technical assistance. This research was supported by US National Science Foundation grant MCB-023499I, and by a grant from the University of Massachusetts Healey Endowment.

\section{References}

I. Edlund T, Normark S: Recombination between short DNA homologies causes tandem duplication. Nature 1981, 292:269-27I.

2. Edlund T, Grundstrom T, Bjork GR, Normark S: Tandem duplication induced by an unusual ampAl-, ampC-transducing lambda phage: a probe to initiate gene amplification. Mol Gen Genetics 1980, I 80:249-257.

3. Andersson RP, Roth JR: Tandem genetic duplication in phage and bacteria. Annu Rev Microbiol 1977, 3 I:473-505.

4. Tlsty TD, Albertini AM, Miller JH: Gene amplification in the lac region of E. coli. Cell 1984, 37:217-224.

5. Rosenberg SM, Longerich S, Gee P, Harris RS: Adaptive mutation by deletions in small mononucleotide repeats. Science 1994, 265:405-407.

6. Foster PL, Trimarchi JM: Adaptive reversion of a frameshift mutation in Escherichia coli by simple base deletions in homopolymeric runs. Science 1994, 265:407-409.

7. Foster PL: Population dynamics of a Lac- strain of Escherichia coli during selection for lactose utilization. Genetics 1994, I38:253-26I.

8. Hastings PJ, Bull HJ, Klump JR, Rosenberg SM: Adaptive amplification: an inducible chromosomal instability mechanism. Cell 2000, I03:723-731.

9. Slechta ES, Bunny KL, Kugelberg E, Kofoid E, Andersson DI, Roth JR: Adaptive mutation: general mutagenesis is not a programmed response to stress but results from rare coamplification of dinB with lac. Proc Natl Acad Sci USA 2003, I 00: | 2847-| 2852 .

10. Slack A, Thornton PC, Magner DB, Rosenberg SM, Hastings PJ: On the mechanism of gene amplification induced under stress in Escherichia coli. PLOS Genetics 2006, 2:e48.

II. Morag AS, Saveson CJ, Lovett ST: Expansion of DNA repeats in Escherichia coli : effects of recombination and replication functions. J Mol Biol 1999, 289:21-27.
12. Foster PL: Adaptive mutation in Escherichia coli. J Bacteriol 2004, 186:4846-4852

13. Cairns J, Foster PL: Adaptive reversion of a frameshift mutation in Escherichia coli. Genetics I99I, I 28:695-70I.

14. Lloyd RG, Low KB: Homologous recombination. In Escherichia coli and Salmonella: Cellular and Molecular Biology 2nd edition. Edited by: Neidhardt FC. ASM Press; 1996:2236-2255.

15. Dutra BE, Sutera VA, Lovett ST: RecA-independent recombination is efficient but limited by exonucleases. Proc Natl Acad Sci USA 2007, 104:216-22I.

16. Lloyd RG: Conjugational recombination in resolvase-deficient ruvC mutants of Escherichia coli K-12 depends on recG. J Bacteriol I99|, |73:54|4-54I8.

17. Khairnar NP, Kamble VA, Mangoli SH, Apte SK, Misra HS: Involvement of a periplasmic protein kinase in DNA strand break repair and homologous recombination in Escherichia coli. Mol Microbiol 2007, 65:294-304.

18. Vuong P, Bennion D, Mantei J, Frost D, Misra R: Analysis of YfgL and $Y$ aeT interactions through bioinformatics, mutagenesis, and biochemistry. J Bacteriol 2008, 190:1507-1517.

19. Steiner P, Sauer U: Overexpression of the ATP-dependent helicase RecG improves resistance to weak organic acids in Escherichia coli. Appl Microbiol Biotechnol 2003, 63:293-299.

20. Zieg J, Maples VR, Kushner SR: Recombination levels of Escherichia coli K-I 2 mutants deficient in various replication, recombination, or repair genes. J Bacteriol 1978, 134:958-966.

21. Wang TV, Smith KC: Inviability of dam recB cells of Escherichia coli is correlated with their inability to repair DNA doublestrand breaks produced by mismatch repair. J Bacteriol 1986, 165:1023-1025.

22. Nowosielska A, Marinus MG: Cisplatin induces DNA doublestrand break formation in Escherichia coli dam mutants. DNA Repair 2005, 7:773-78I.

23. Lombardo M, Aponyi I, Rosenberg SM: General stress response regulator RpoS in adaptive mutation and amplification in Escherichia coli. Genetics 2004, 166:669-680.

24. Poteete AR, Fenton AC: Genetic requirements of phage $\lambda$ Redmediated gene replacement in Escherichia coli K-12. J Bacteriol 2000, 182:2336-2340.

25. Konrad EB: Method for the isolation of Escherichia coli mutants with enhanced recombination between chromosomal duplications. J Bacteriol 1977, 130:167-172.

26. Blakely GW, Murray NE: Control of the endonuclease activity of type I restriction-modification systems is required to maintain chromosome integrity following homologous recombination. Mol Microbiol 2006, 60:883-893.

27. Stahl FW, McMilin KD, Stahl MM, Craseman JM, Lam S: The distribution of crossovers along unreplicated lambda bacteriophage chromosomes. Genetics 1974, 77:395-408.

28. Stahl FW, McMilin KD, Stahl MM, Nozu Y: An enhancing role for DNA synthesis in formation of bacteriophage $\lambda$ recombinants. Proc Natl Acad Sci USA 1972, 69:3598-360I.

29. Stahl MM, Thomason L, Poteete AR, Tarkowski T, Kuzminov A, Stahl $\mathrm{FW}$ : Annealing vs. invasion in phage $\lambda$ recombination. Genetics 1997, 147:96|-977.

30. Poteete AR: Involvement of DNA replication in phage lambda Red-mediated homologous recombination. Mol Microbiol 2008, 68:66-74.

31. Court DL, Sawitzke JA, Thomason LC: Genetic engineering using homologous recombination. Annu Rev Genet 2002, 36:36I-388.

32. deMassy B, Fayet $O$, Kogoma T: Multiple origin usage for DNA replication in sdrA (rnhA) mutants of Escherichia coli K-I 2: initiation in the absence of oriC. J Mol Biol 1984, 178:227-236.

33. Boye $E$, Løbner-Olesen $A$ : The role of dam methyltransferase in the control of DNA replication in E. coli. Cell I990, 62:98I-989.

34. Kogoma T, Hong X, Caldwell GW, Barnard KG, Asai T: Requirement of homologous recombination functions for viability of the Escherichis coli cell that lacks RNase $\mathrm{HI}$ and exonuclease V activities. Biochimie 1993, 75:89-99.

35. Marinus MG, Morris NR: Biological function for 6-methyladenine residues in the DNA of Escherichia coli KI2. J Mol Biol 1974, 85:309-322.

36. Poteete AR, Fenton AC, Nadkarni A: Chromosomal duplications and cointegrates generated by the bacteriophage lambda 
Red system in Escherichia coli K-I 2. BMC Molecular Biology 2004, 5:22.

37. Stumpf JD, Poteete AR, Foster PL: Amplification of lac cannot account for adaptive mutation to Lac+ in Escherichia coli. J Bacteriol 2007, 189(6):2291-2299.

38. Rosche WA, Foster PL: The role of transient hypermutators in adaptive mutation in Escherichia coli. Proc Natl Acad Sci USA 1999, 96:6862-6867.

39. Baba T, Ara T, Hasegawa M, Takai Y, Okumura Y, Baba M, Datsenko KA, Tomita M, Wanner BL, Mori H: Construction of Escherichia coli K-I 2 in-frame, single-gene knockout mutants: the Keio collection. Mol Syst Biol 2006, 2:2006.0008.

40. Murphy KC, Campellone KG, Poteete AR: PCR-mediated gene replacement in Escherichia coli. Gene 2000, 246:32 I-330.

41. Poteete AR, Fenton AC, Murphy KC: Roles of RuvC and RecG in phage $\lambda$ Red-mediated recombination. J Bacteriol 1999, 181:5402-5408.

42. Poteete $A R$, Fenton AC, Wang HR: Recombination-promoting activity of the bacteriophage $\lambda$ Rap Protein in Escherichia coli K-I 2. J Bacteriol 2002, 184:4626-4629.

43. Poteete AR: Modulation of DNA repair and recombination by the bacteriophage $\lambda$ Orf function in Escherichia coli $K-12$. J Bacteriol 2004, I 86:2699-2707.

44. Poteete AR, Rosadini C, Pierre CS: Gentamicin and other cassettes for chromosomal gene replacement in Escherichia coli. Biotechniques 2006, 4I(3):26I-263.

45. Murphy KC, Campellone KG: Lambda Red-mediated recombinogenic engineering of enterohemorrhagic and enteropathogenic E. coli. BMC Molecular Biology 2003, 4: I I.

46. Uhlin BE, Volkert MR, Clark AJ, Sancar A, Rupp WD: Nucleotide sequence of a recA operator mutation. Mol Gen Genet 1982 I 85:25I-254.

Publish with Bio Med Central and every scientist can read your work free of charge

"BioMed Central will be the most significant development for disseminating the results of biomedical research in our lifetime. "

Sir Paul Nurse, Cancer Research UK

Your research papers will be:

- available free of charge to the entire biomedical community

- peer reviewed and published immediately upon acceptance

- cited in PubMed and archived on PubMed Central

- yours - you keep the copyright 